\title{
Alternative healthcare with modern medicine
}

\section{Opinion}

Modern medicine uses the approach of treating the numbers, lab results, chext x-rays, Ct Scanns. As a veteran of the respiratory field for 27years. I have come to the conclusion that patients need more than just a medication adjustment for successful illness management. Treating the patient as a whole in conjunction mentally, spiritually along with physical symptoms is crucial for recovery. The quick fix seems to be the flow of treatment instead of treating the root problem. Mostly due to staffing shortages and large patient to health care professional ratio. The scientific community goes more on treatment of numbers than things that are subjective. Human beings as a whole need emotional, spiritual and physical support in order to heal. As a respiratory practitioner of I have been on both sides of the bed. One as a daughter, mother and I myself have been on the receiving end of medical care.

The treatment of Chronic Lung patients or any patient that presents with acute or chronic shortness of breath. Has the obstacle of overcoming the severe anxiety that is associated with the feeling of dyspnea. Although, their Abg results could show completely normal results the feeling still needs to be treated. Just the use of bronchodilator therapy along with steriods does not always produce the results of decreasing the patient's discomfort. Therefore, requiring anywhere from continuous nebulisation to sedation and intubation. Majority of the patient's intubations could have been prevented with earlier intervention and management of their anxiety. Which would decrease hospital admission time and costs?

Sure, we use bronchodilator therapy, bronchial hygiene, steroid therapy and controlled substances such as ativan and xanax for symptom management. Bipap is then initiated if symptoms are not controlled. These modalities are even implemented on CHF, Cancer and pneumonia patients. What if, there was an alternative preventative therapy that helped decrease the anxiety level that some of these patients didn't need more invasive treatments. Over 800 hospitals throughout the United States are using the holistic approach of Reiki in their regular care in the Intensive Care Units and Surgical Units. It is being implemented for use of decreased anxiety and pain control. Hospices are using Reiki for palliative care for patient comfort and transition at end of life. Reiki has shown an improvement in recovery rate post op and the decrease of unwanted side effects of medications such as chemotherapy. Small controlled studies have shown an improvement in psychological effects of depression and anxiety.

Minimal research has been done on the use of Reiki and severe dyspnea in lung patients. Most of the patients I have dealt with over the years have the constant "feeling" of dyspnea and high anxiety. In some patients I have cared for controlled substances have not done the job of decreasing the symptoms. Intubation with diprovan or precedex has been required in order for respiratory status to be stabilized. In severe cases I am not indicating that Reiki would prevent intubation. Reiki may provide improvement in weaning in mechanical ventilation in more severe anxiety cases...causing less use of controlled substances. Reiki provides a sense of calm, relaxation and well being. The use of alternative therapies is becoming more popular and sought out in the public communities today.

Research that I found on controlled studies using Reiki along with other therapy under Choen's statistic showed: 0.24 decrease in anxiety
Volume 2 Issue 3 - 2015

\author{
Cauleen Denman \\ Central Regional Medical Center, USA
}

Correspondence: Cauleen Denman, Central Regional Medical Center, 35442 N Treasure Island Blvd Leesburg, FI 34788, USA, Tel 407-274-0868, Email denmancauleen@yahoo.com

Received: February 19, 2015 | Published: March 19, 2015

in patients with anxiety and 2.09 for pain in adults with surgical procedures. When used in cancer patient's vs rest intervention a 4.5 decrease in anxiety and pain. Other small studies have shown that Reiki along with regular medical treatment does help in severe treatment of pain and anxiety. The respiratory practitioner is at bedside with acute cases. Why not implement reiki to decrease anxiety and increase level of well being. Qualities studies have shown that pts have a feeling of being more relaxed, safe, secure and dreamy after having a reiki session. Of course, it could not be used in emergent cases.

Our body produces a measurable bio energy field. The heart, brain and soft tissues all have electrical fields that can be monitored. In 1990 a Japanese team measured that 0.3 to 30 hertz cycles was measure from the hands of a person performing reiki. These varied pulses have been shown to be effective for stroke, bone stimulation and many other medical diagnoses. If, Reiki is able to decrease the anxiety, work of breathing and improve a lung patient's comfort and quality of life. Why not implement it into their plan of care. It could be a new way of treating patients that decrease hospital admissions and stays. Reiki along with good pulmonary hygiene and management would be treating the patient as a whole.

Other allied healthcare practioners such as nurses and Arnp's are now implementing Reiki into their regular practice. Reiki can also be used on yourself once attuned to it. Patients who are able to be attuned could provide their own preventative care at home along with their bronchodilator and steroid therapy. Allied healthcare professional also could use on their self for stress management and burn out. Nurses who have used stated that it did decrease their stress level and they felt more grounded in providing patient care after a Reiki session.

Hopefully, there will be more in depth research and studies done in the future. It would be an improvement to see the patient treated as a whole and not just by the lab results.

\section{Acknowledgements}

None.

\section{Conflict of interest}

The author declares no conflict of interest. 